\title{
Oscillatory motion of a counterpropagating Kerr soliton dimer
}

\author{
Chengying Bao, ${ }^{1}$ Boqiang Shen, ${ }^{1}$ Myoung-Gyun Suh, ${ }^{1, *}$ Heming Wang, ${ }^{1}$ Kemal Şafak, ${ }^{2}$ Anan Dai, ${ }^{2}$ \\ Andrey B. Matsko, ${ }^{3}$ Franz X. Kärtner, ${ }^{4,5}$ and Kerry Vahala $\oplus^{1,+}$ \\ ${ }^{1}$ T. J. Watson Laboratory of Applied Physics, California Institute of Technology, Pasadena, California 91125, USA \\ ${ }^{2}$ Cycle GmbH, 22607 Hamburg, Germany \\ ${ }^{3}$ Jet Propulsion Laboratory, California Institute of Technology, Pasadena, California 91109, USA \\ ${ }^{4}$ Center for Free-Electron Laser Science, Deutsches Elektronen-Synchrotron, 22607 Hamburg, Germany \\ ${ }^{5}$ Department of Physics and the Hamburg Center for Ultrafast Imaging, University of Hamburg, 22761 Hamburg, Germany
}

(Received 25 February 2020; revised 15 December 2020; accepted 18 December 2020; published 6 January 2021)

\begin{abstract}
Counterpropagating (CP) solitons generated in high- $Q$ microcavities not only offer useful dual-comb sources, but also provide a new platform to study soliton interactions. Here, we demonstrate and theoretically explain a manifestation of soliton trapping that occurs between CP solitons in a silica microcavity introducing a Kerr soliton dimer. In conventional soliton trapping, the group velocities of two solitons can be synchronized by a Kerr-effect-mediated interaction. The solitons can then copropagate with a fixed temporal delay. However, as shown here, when counterpumping a microcavity using slightly detuned pump frequencies and in the presence of backscattering, the group velocities of clockwise and counterclockwise solitons undergo periodic modulation instead of being locked to a constant velocity. Upon emission from the microcavity, the solitons feature a relative oscillatory motion around a locked average relative displacement with an amplitude that can be larger than the soliton pulse width. This relative motion introduces a sideband fine structure into the optical spectrum of the $\mathrm{CP}$ solitons. Our observation provides insights on coherently pumped soliton dimers in microcavities.
\end{abstract}

DOI: 10.1103/PhysRevA.103.L011501

Coherently pumped optical solitons were first studied within the subject of spatial cavity solitons [1-4]. Their counterparts, coherently pumped temporal solitons, have also been demonstrated in passive fiber cavities [5] and high- $Q$ microcavities [6-10]. And the realization of such microcavity solitons represents a significant advance of microresonator-based Kerr frequency combs (microcombs) [10,11]. These soliton microcombs can pave the way towards compact spectrometers [12], Lidar systems [13,14], optical frequency synthesizers [15], and optical clocks [16]. The advent of microcavity solitons and their mathematical relationship to various multiparticle physical systems also make these systems useful for improved understanding of intricate physical systems. For example, they have been utilized to demonstrate soliton crystals [17], heteronuclear soliton molecules [18], and soliton formation in photonic dimers [19]. Furthermore, they provide a platform for the study of Cherenkov radiation [20-24], Fermi-Pasta-Ulam recurrence [25-27], Feshbach resonances [28], and Bose-Hubbard hopping [29-33].

The symmetry of the clockwise (CW) and counterclockwise $(\mathrm{CCW})$ propagation directions enables the generation of counterpropagating (CP) solitons in microcavities [34,35]. Unlike copropagating solitons, CP solitons can interact via Rayleigh backscattering of the microcavity [34], which adds a new element for study of soliton physics. For example, the interaction of CP solitons via a single comb line can stabilize the

\footnotetext{
*Present address: Physics \& Informatics Laboratories, NTT Research, Inc., 940 Stewart Drive, Sunnyvale, California 94085, USA.

†vahala@caltech.edu
}

repetition rate difference $\left(\Delta f_{r}\right)$ between $\mathrm{CP}$ solitons to $\Delta f_{r}=$ $\Delta v_{P} / N$, where $\Delta v_{P}$ is the frequency detuning between the counterpropagating pumping waves (typically several $\mathrm{MHz}$ for silica microcavities) and $N$ is an integer [34]. A repetition rate locking regime has also been observed when $\Delta v_{P}$ is small (e.g., tens or hundreds of $\mathrm{kHz}$ ) and the pump powers are nearly balanced [34-36]. However, the residual soliton motion in this regime (hereinafter referred to as the ' $\Delta f_{r}=0$ regime') has not been revealed due to the insufficient temporal resolution of the measurement methods.

In this paper, we report the measurement of the relative $\mathrm{CP}$ soliton motion in the $\Delta f_{r}=0$ regime using a balanced optical cross-correlator (BOC) [37]. This regime can be understood to arise from the Kerr-mediated soliton trapping effect [38,39] between the $\mathrm{CW}(\mathrm{CCW})$ soliton and the backscattered field from the $\mathrm{CCW}$ (CW) soliton. Surprisingly, an oscillatory motion between CP solitons (after coupling out from the microcavity) at a frequency of $\Delta v_{P}$ is observed. The amplitude of this motion can be larger than the soliton pulse width. The motion makes $\mathrm{CP}$ soliton trapping different from conventional soliton trapping, which locks the interacting solitons to travel at the same group velocity and a fixed time delay. Thus, our work adds a new insight into the understanding of soliton trapping dynamics. Moreover, it establishes a new type of soliton dimer molecule [18] formed by interacting CP solitons.

$\mathrm{CP}$ soliton dimer formation is enabled by the soliton trapping process. This trapping process can arise from the cross-phase-modulation induced refractive index trapping potential, which locks the group velocities of two interacting solitons [38-41]. The pulse phase velocities can also be locked via a four-wave-mixing interaction term [40] when the phase 


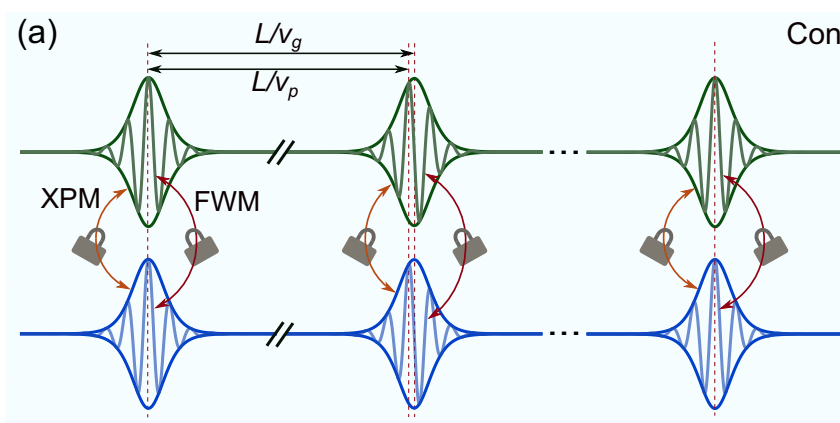

Conventional soliton trapping

(b)

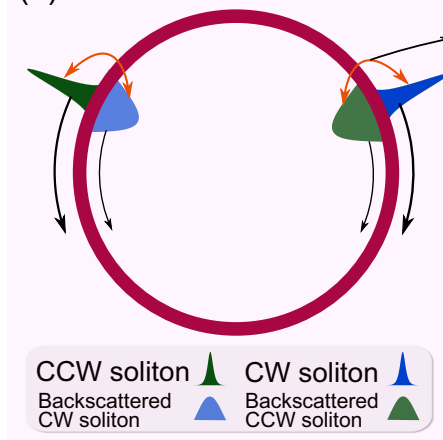

Trapping with forced motion

FIG. 1. Forced motion in a trapped CP soliton dimer. (a) In conventional soliton trapping without coherent pumps, both the phase velocity and the group velocity will be locked. In the frequency domain, both the repetition rate $f_{r}$ and the carrier envelope offset frequency $f_{0}$ of the corresponding combs will be equal. Note that in the illustration the pulse envelopes are plotted to be exactly overlapped and the phase is identical, which are not necessary in experiments. (b) For CP solitons in coherently pumped systems, backscattering enables a different trapping outcome. Due to the coherent pumping, the phase velocity and $f_{0}$ cannot be locked when using nondegenerate pumps. In this case, solitons will be locked in an averaged way, with the spectral center frequency $\left(v_{s}\right)$ and group velocity experiencing periodic modulation (upper shaded box). As a result, there is relative motion between the CP solitons after being emitted from the microcavity (lower shaded box). This relative motion repeats with a period of $1 / \Delta v_{P}$. In the frequency domain, the motion will induce sidebands around the main comb lines. Note that the power ratio between the solitons and the backscattered counterpart is for illustration and does not reflect the actual ratio.

velocity difference is small enough [see Fig. 1(a)]. In this way, both the pulse envelope and the carrier frequency can be synchronized. And, as a result, the corresponding comb frequencies for each pulse train become identical [see Fig. 1(a)]. The trapping process (in both the group and the phase velocity versions) has also been reported for solitons in coherently pumped fiber and microcavities [17,42-46].

Unavoidable backscattering within a microcavity couples the soliton field into the other propagation direction [34]. This provides a way for each soliton to interact with a modified replica of the other soliton [see Fig. 1(b)]. Because backscattering is not expected to occur at a single point, but rather over a complex spatial profile, the backscattered field itself reflects the complexities of this scattering process and may no longer be a short solitonlike sech pulse. Let us consider first the case of degenerate pumping frequencies $\left(\Delta v_{P}=0\right)$. CCW and $\mathrm{CW}$ solitons share the same longitudinal mode family and similar pump conditions (same pump frequency and similar pump power). Moreover, a feature of the coherent pumping is that the pump frequency is one of the soliton comb frequencies. For these reasons both the envelope and the carrier of CP solitons will have closely matched velocities. Moreover, the Kerreffect-mediated interaction allows the two solitons to trap one another via the backscattered fields in a way very similar to the trapping of conventional copropagating solitons [38-40]. Thus, even while the solitons are propagating in opposite directions, their group and phase velocities become locked.
When the pump frequency detuning $\Delta v_{P}$ is nonzero but small compared to the cavity linewidth, we show that the CP solitons are still bound to each other. However, on account of the coherent nature of the pumping, the frequency of each soliton comb line is shifted by $\Delta v_{P}$ relative to the replica of the other soliton comb. Importantly, this frequency shift is set by the external pumps and cannot be pulled towards 0 as in conventional soliton trapping [Fig. 1(b)]. The soliton carrier and phase velocities are therefore not synchronized. Thus, each of the backscattered CW (CCW) comb lines can be regarded as a sideband that modulates the $\mathrm{CCW}(\mathrm{CW})$ comb lines [see upper gray-shaded box in Fig. 1(b)]. This modulation causes each soliton to experience a periodic spectral center frequency change $\left(\Delta v_{s}\right)$, with the period being $1 / \Delta v_{P}$. The group velocity of the pulses varies with the spectral center as $\Delta\left(1 / v_{g}\right)=2 \pi \beta_{2} \Delta v_{s}$, where $\beta_{2}$ is the group velocity dispersion (negative for cavities supporting solitons) [47,48]. This group velocity modulation causes periodic relative motion of $\mathrm{CP}$ solitons, with a period of $1 / \Delta v_{P}$ within their traps. Corresponding frequency sidebands spaced by $\Delta v_{P}$ emerge around the main comb lines [see right panel in Fig. 1(b)].

We used the experimental setup shown in Fig. 2(a) to measure the predicted motion. $\mathrm{CP}$ solitons were generated in a $22-\mathrm{GHz}$ high- $Q$ silica wedge microcavity $[7,34,49]$. A single pump laser was used for generation of $\mathrm{CP}$ solitons and distinct pumping frequencies for $\mathrm{CW}$ and $\mathrm{CCW}$ directions 
(a)

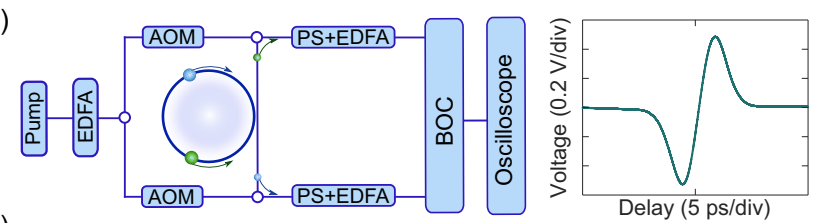

(b)

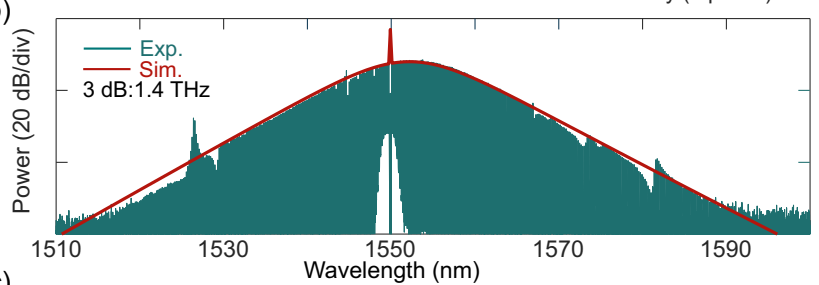

(c)

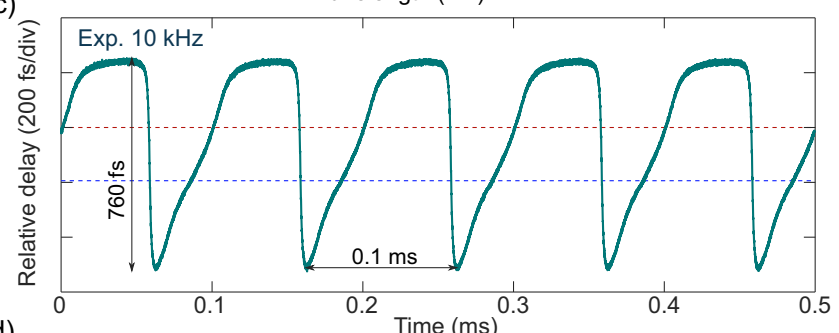

(d)

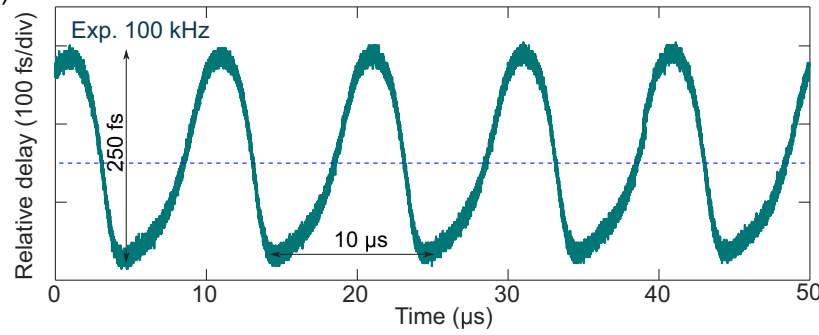

FIG. 2. Observation of oscillatory motion in CP soliton trapping. (a) A balanced optical cross correlator (BOC) is used to experimentally measure relative $\mathrm{CP}$ soliton motion (left). The BOC output signal is shown when the delay is scanned (right). AOM, acoustooptical modulator; EDFA, erbium-doped fiber amplifier; PS, pulse shaper. (b) Optical spectrum of the CP solitons with a 3-dB bandwidth of $1.4 \mathrm{THz}$. The red line (spectral envelope) is the simulated spectrum. (c), (d) BOC-measured relative soliton motion when the pump frequency detuning is 10 and $100 \mathrm{kHz}$, respectively. The motion frequency is measured to be equal to $\Delta v_{P}$. The dashed red (upper) and blue (lower) lines indicate the center of motion for 10and $100-\mathrm{kHz}$ detuning, respectively; and they are shifted by about $200 \mathrm{fs}$ as shown in (c).

were produced by two acousto-optical modulators [34]. The corresponding optical spectrum of one of the $\mathrm{CP}$ solitons is shown in Fig. 2(b) and has a 3-dB bandwidth of $1.4 \mathrm{THz}$ so that the soliton duration is deduced to be 125 fs [equivalently, 220 fs for the full width at half-maximum (FWHM) pulse width]. We used a BOC to record the soliton motion, which operates by balanced detection of the sum frequency generation between two inputs in a PPKTP crystal [37]. Before input into the BOC, the soliton streams (with pumps suppressed by notch filters) were dispersion compensated by pulse shapers [50] and amplified, so as to enhance the BOC output signal. Figure 2(a) also shows an example of the BOC output signal when the delay line inside the BOC was scanned. The central portion of the signal is linear. Thus, when setting the delay line in that region, soliton motion can be converted into a voltage signal for measurements.
The output of the BOC for pump detuning frequencies of 10 and $100 \mathrm{kHz}$ is shown in Figs. 2(c) and 2(d). These resulting temporal modulation rates are much lower than the BOC detection bandwidth of $4 \mathrm{MHz}$. The ability to reliably observe a nonzero signal implies that the repetition rates of the two solitons are locked on average (i.e., operating in the $\Delta f_{r}=0$ regime), since otherwise the two inputs would temporally walk off, resulting in a zero signal due to nonsynchronized repetition rates [37]. A strong oscillation of the BOC signal is observed, with a peak-to-peak amplitude reaching $0.8 \mathrm{ps}$, which is more than twice the soliton FWHM pulse width. This is also nearly $2 \%$ of the round-trip time (46 ps). The motion is not sinusoidal but is asymmetric (sawtoothlike in the $10-\mathrm{kHz}$ detuning case). And the oscillation frequency is equal to the pump frequency detuning $\left(\Delta v_{P}\right)$ as expected. The center of mass of the relative motion trajectory is also plotted in Figs. 2(c) and 2(d). It suggests that the CP solitons oscillate around different centers in the trap when the pump detuning frequency varies. This motion exists for small detunings, e.g., $\Delta v_{P}<1 \mathrm{~Hz}$, suggesting that the solitons interact on an ultralong time scale. The noisier trace in Fig. 2(d) compared to Fig. 2(c) is under investigation but could result from the Raman process. Specifically, the Raman-induced soliton frequency shift has a quadratic dependence on pump resonance detuning [51], and hence the relative group velocity of $\mathrm{CP}$ solitons is more susceptible to pump-resonance-detuning fluctuations when $\Delta v_{P}$ is larger.

Numerical simulations based on the coupled LugiatoLefever equations $[1,34,52,53]$ confirm the experimental observations [54]. Representative plots of the relative soliton motion are shown in Figs. 3(a) and 3(b). The motion frequency equals $\Delta v_{P}$, and both the trajectory and the amplitude are reasonably consistent with the experimental measurements. For example, the asymmetric sawtoothlike behavior is numerically reproduced. The simulation assumed a single-point backscatterer, while there are likely multiple backscatterering centers in the actual microcavity. It is therefore expected that some discrepancies exist in the observed and simulated motion trajectories. More discussion of the asymmetric motion can be found in [54].

To test the hypothesis that the relative motion is driven by the detuned-pump-induced forced-soliton spectral center shift, we numerically calculated the relative spectral center frequency between the two solitons in Fig. 3(c). It exhibits periodic oscillation around zero frequency. The positive and negative relative frequency regions correspond to forced motion where the derivative of the relative delay (i.e., relative group velocity) is positive or negative, respectively [see vertical dashed green lines in Figs. 3(b) and 3(c)]. Moreover, the fact that the relative center frequency oscillates around $0 \mathrm{~Hz}$ indicates that their group velocities are locked on average. Accordingly, the experimental and numerical observations validate the existence of oscillatory forced motion of the $\mathrm{CP}$ soliton dimer in the presence of backscattering.

Finally, we experimentally verify that the forced motion introduces fine-structure sidebands into the comb lines. For this measurement, the two $\mathrm{CP}$ soliton microcombs are heterodyned on a balanced photodetector. The recorded electrical spectra, shown in Fig. 4, contain multiple radio-frequency tones for pump detuning of both 10 and $100 \mathrm{kHz}$. The lack 
(a)

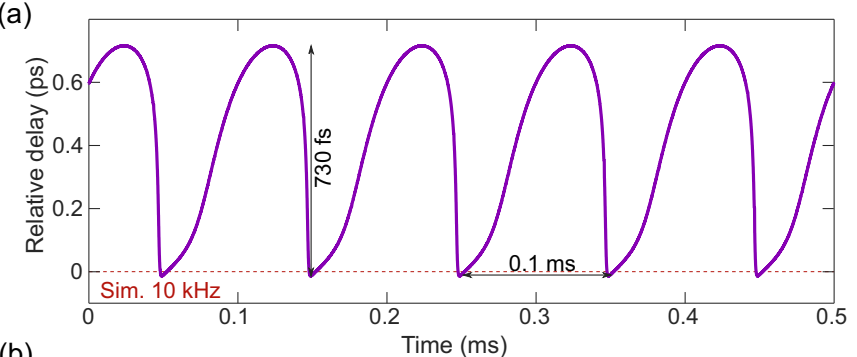

(b)
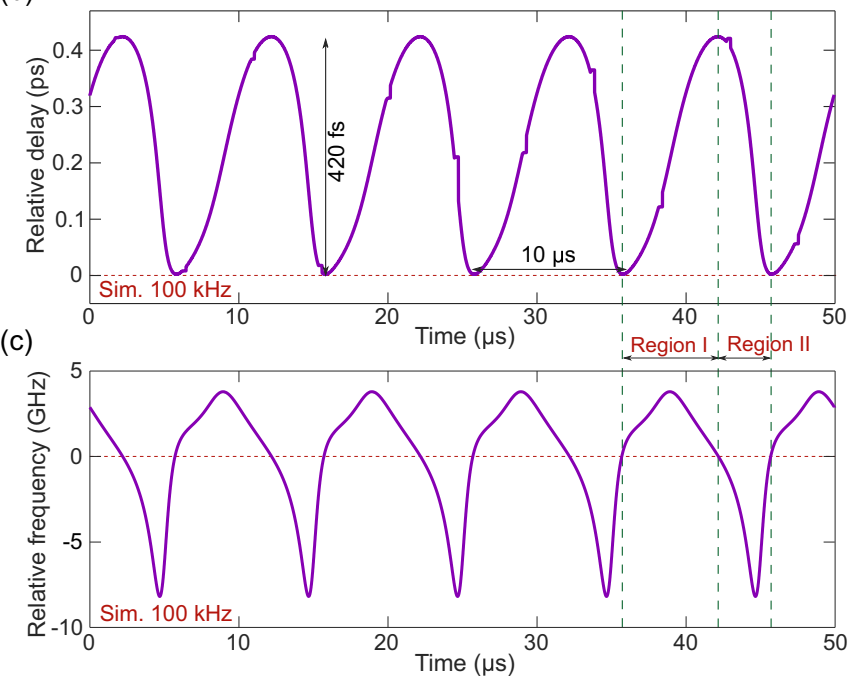

FIG. 3. Simulation of forced motion in CP soliton trapping. (a), (b) Simulated relative soliton temporal motion when the two pumps are detuned by 10 and $100 \mathrm{kHz}$, respectively. Dashed red lines indicate the zero delay. (c) Numerically calculated relative spectral center frequency between two CP solitons for $\Delta v_{P}=100 \mathrm{kHz}$, showing periodic variation. Depending upon the sign of the relative frequency, the motion can be separated into two regions as indicated by the dashed green lines.

of any tone other than those at integer multiples of $\Delta v_{P}$ shows that only fine-structure sidebands spaced by $\Delta v_{P}$ are present in the optical comb spectra. Sidebands due to soliton spectral bandwidth breathing induced by Rayleigh backscattering in a microcavity were also simulated in Ref. [32]. Different from that instability, the measured sidebands here arise from the forced relative soliton motion.

In summary, we have measured the relative oscillatory motion between $\mathrm{CP}$ solitons in the $\Delta f_{r}=0$ regime. This temporal motion results from slightly detuned coherent (a)

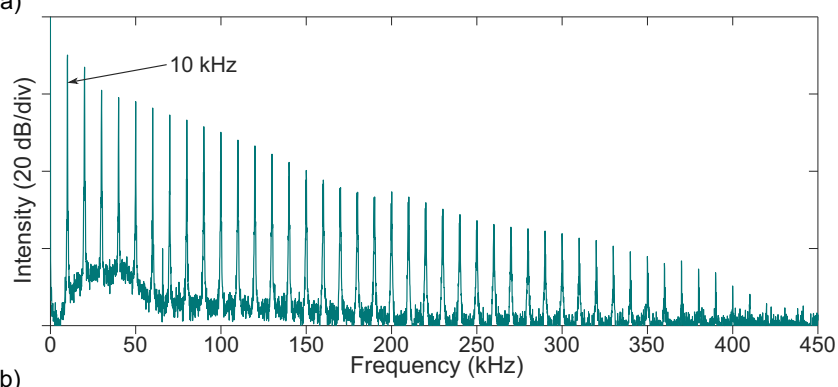

(b)

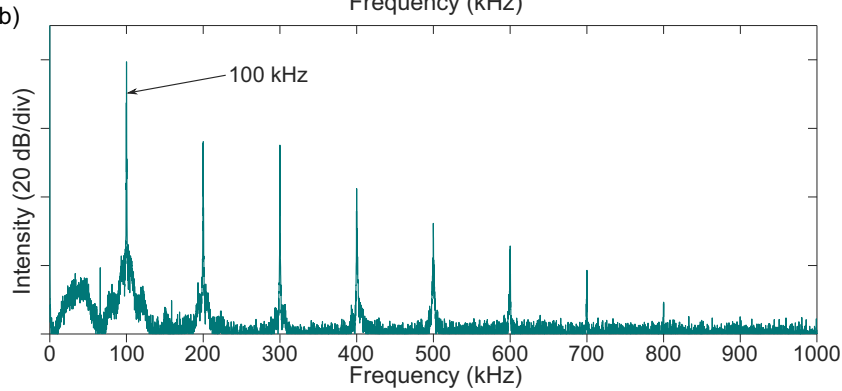

FIG. 4. Measured electrical spectra of the beat between CP solitons. (a) $\Delta v_{P}=10 \mathrm{kHz}$. (b) $\Delta v_{P}=100 \mathrm{kHz}$.

counterpumping of $\mathrm{CP}$ solitons in the presence of optical backscattering. The detuned counterpumps cause a periodic modulation of both the spectral center frequencies of $\mathrm{CP}$ solitons and their relative group velocities. Different from the $\Delta f_{r}=\Delta v_{P} / N$ regime, all comb lines participate in the soliton interaction in the $\Delta f_{r}=0$ regime. Our measurements show that it does not require the solitons to be constrained within a small temporal range for trapping of solitons and how coherently pumped solitons are different from conservative solitons. The oscillatory motion also inserts fine-structure sidebands into the soliton microcomb spectrum that may affect some comb applications. The results provide new insights and generalize somewhat the concept of a soliton dimer in microcavities.

This work was supported by the Air Force Office of Scientific Research (Grant No. FA9550-18-1-0353) and the Kavli Nanoscience Institute. C.B. gratefully acknowledges the postdoctoral fellowship from the Resnick Sustainability Institute at Caltech. The research performed by A.M. was carried out at the Jet Propulsion Laboratory, California Institute of Technology, under a contract with the National Aeronautics and Space Administration (No. 80NM0018D0004). We thank Qi-Fan Yang for helpful discussion.
[1] L. A. Lugiato and R. Lefever, Spatial Dissipative Structures in Passive Optical Systems, Phys. Rev. Lett. 58, 2209 (1987).

[2] W. J. Firth and A. J. Scroggie, Optical Bullet Holes: Robust Controllable Localized States of a Nonlinear Cavity, Phys. Rev. Lett. 76, 1623 (1996).

[3] S. Barland, J. R. Tredicce, M. Brambilla, L. A. Lugiato, S. Balle, M. Giudici, T. Maggipinto, L. Spinelli, G. Tissoni, T. Knoedl et al., Cavity solitons as pixels in semiconductor microcavities, Nature 419, 699 (2002).
[4] T. Ackemann, W. J. Firth, and G.-L. Oppo, Fundamentals and applications of spatial dissipative solitons in photonic devices, Adv. At. Mol. Opt. Phys. 57, 323 (2009).

[5] F. Leo, S. Coen, P. Kockaert, S.-P. Gorza, P. Emplit, and M. Haelterman, Temporal cavity solitons in one-dimensional Kerr media as bits in an all-optical buffer, Nat. Photon. 4, 471 (2010).

[6] T. Herr, V. Brasch, J. D. Jost, C. Y. Wang, N. M. Kondratiev, M. L. Gorodetsky, and T. J. Kippenberg, Temporal solitons in optical microresonators, Nat. Photon. 8, 145 (2014). 
[7] X. Yi, Q.-F. Yang, K. Y. Yang, M.-G. Suh, and K. Vahala, Soliton frequency comb at microwave rates in a high-Q silica microresonator, Optica 2, 1078 (2015).

[8] C. Joshi, J. K. Jang, K. Luke, X. Ji, S. A. Miller, A. Klenner, Y. Okawachi, M. Lipson, and A. L. Gaeta, Thermally controlled comb generation and soliton modelocking in microresonators, Opt. Lett. 41, 2565 (2016).

[9] P.-H. Wang, J. A. Jaramillo-Villegas, Y. Xuan, X. Xue, C. Bao, D. E. Leaird, M. Qi, and A. M. Weiner, Intracavity characterization of micro-comb generation in the single-soliton regime, Opt. Express 24, 10890 (2016).

[10] T. J. Kippenberg, A. L. Gaeta, M. Lipson, and M. L. Gorodetsky, Dissipative Kerr solitons in optical microresonators, Science 361, eaan8083 (2018).

[11] P. Del'Haye, A. Schliesser, O. Arcizet, T. Wilken, R. Holzwarth, and T. J. Kippenberg, Optical frequency comb generation from a monolithic microresonator, Nature 450, 1214 (2007).

[12] M.-G. Suh, Q.-F. Yang, K. Y. Yang, X. Yi, and K. J. Vahala, Microresonator soliton dual-comb spectroscopy, Science 354, 600 (2016).

[13] M.-G. Suh and K. J. Vahala, Soliton microcomb range measurement, Science 359, 884 (2018).

[14] P. Trocha, M. Karpov, D. Ganin, M. Pfeiffer, A. Kordts, S. Wolf, J. Krockenberger, P. Marin-Palomo, C. Weimann, S. Randel et al., Ultrafast optical ranging using microresonator soliton frequency combs, Science 359, 887 (2018).

[15] D. T. Spencer, T. Drake, T. C. Briles, J. Stone, L. C. Sinclair, C. Fredrick, Q. Li, D. Westly, B. R. Ilic, A. Bluestone et al., An integrated-photonics optical-frequency synthesizer, Nature 557, 81 (2018).

[16] Z. L. Newman, V. Maurice, T. Drake, J. R. Stone, T. C. Briles, D. T. Spencer, C. Fredrick, Q. Li, D. Westly, B. R. Ilic et al., Architecture for the photonic integration of an optical atomic clock, Optica 6, 680 (2019).

[17] D. C. Cole, E. S. Lamb, P. Del'Haye, S. A. Diddams, and S. B. Papp, Soliton crystals in Kerr resonators, Nat. Photon. 11, 671 (2017).

[18] W. Weng, R. Bouchand, E. Lucas, E. Obrzud, T. Herr, and T. J. Kippenberg, Heteronuclear soliton molecules in optical microresonators, Nat. Commun. 11, 2402 (2020).

[19] A. Tikan, J. Riemensberger, K. Komagata, S. Hönl, M. Churaev, C. Skehan, H. Guo, R. N. Wang, J. Liu, P. Seidler et al., Emergent nonlinear phenomena in a driven dissipative photonic dimer, arXiv:2005.06470.

[20] P. K. A. Wai, C. R. Menyuk, Y. C. Lee, and H. H. Chen, Nonlinear pulse propagation in the neighborhood of the zerodispersion wavelength of monomode optical fibers, Opt. Lett. 11, 464 (1986).

[21] N. Akhmediev and M. Karlsson, Cherenkov radiation emitted by solitons in optical fibers, Phys. Rev. A 51, 2602 (1995).

[22] V. Brasch, M. Geiselmann, T. Herr, G. Lihachev, M. H. P. Pfeiffer, M. L. Gorodetsky, and T. J. Kippenberg, Photonic chip-based optical frequency comb using soliton Cherenkov radiation, Science 351, 357 (2016).

[23] Q.-F. Yang, X. Yi, K. Y. Yang, and K. Vahala, Spatial-modeinteraction-induced dispersive waves and their active tuning in microresonators, Optica 3, 1132 (2016).
[24] A. B. Matsko, W. Liang, A. A. Savchenkov, D. Eliyahu, and L. Maleki, Optical Cherenkov radiation in overmoded microresonators, Opt. Lett. 41, 2907 (2016).

[25] E. Fermi, J. Pasta, and S. Ulam, Los Alamos Report LA-1940 (Los Alamos National Laboratory, Los Alamos, NM, 1955).

[26] A. B. Matsko, A. A. Savchenkov, and L. Maleki, On excitation of breather solitons in an optical microresonator, Opt. Lett. 37, 4856 (2012).

[27] C. Bao, J. A. Jaramillo-Villegas, Y. Xuan, D. E. Leaird, M. Qi, and A. M. Weiner, Observation of Fermi-Pasta-Ulam Recurrence Induced by Breather Solitons in an Optical Microresonator, Phys. Rev. Lett. 117, 163901 (2016).

[28] A. B. Matsko and L. Maleki, Feshbach resonances in Kerr frequency combs, Phys. Rev. A 91, 013831 (2015).

[29] D. Gerace, H. E. Türeci, A. Imamoglu, V. Giovannetti, and R. Fazio, The quantum-optical Josephson interferometer, Nat. Phys. 5, 281 (2009).

[30] T. C. H. Liew and V. Savona, Single Photons from Coupled Quantum Modes, Phys. Rev. Lett. 104, 183601 (2010).

[31] C. Eichler, Y. Salathe, J. Mlynek, S. Schmidt, and A. Wallraff, Quantum-Limited Amplification and Entanglement in Coupled Nonlinear Resonators, Phys. Rev. Lett. 113, 110502 (2014).

[32] A. B. Matsko and L. Maleki, Bose-Hubbard hopping due to resonant Rayleigh scattering, Opt. Lett. 42, 4764 (2017).

[33] B. Krauskopf, N. G. R. Broderick, J. A. Levenson, A. M. Yacomotti, and A. Giraldo, The driven-dissipative BoseHubbard dimer: Phase diagram and chaos, New J. Phys. 22, 043009 (2020).

[34] Q.-F. Yang, X. Yi, K. Y. Yang, and K. Vahala, Counterpropagating solitons in microresonators, Nat. Photon. 11, 560 (2017).

[35] C. Joshi, A. Klenner, Y. Okawachi, M. Yu, K. Luke, X. Ji, M. Lipson, and A. L. Gaeta, Counter-rotating cavity solitons in a silicon nitride microresonator, Opt. Lett. 43, 547 (2018).

[36] W. Weng, R. Bouchand, E. Lucas, and T. J. Kippenberg, Polychromatic Cherenkov Radiation Induced Group Velocity Symmetry Breaking in Counterpropagating Dissipative Kerr Solitons, Phys. Rev. Lett. 123, 253902 (2019).

[37] J. Kim and F. X. Kärtner, Attosecond-precision ultrafast photonics, Laser Photon. Rev. 4, 432 (2010).

[38] C. R. Menyuk, Stability of solitons in birefringent optical fibers. I: Equal propagation amplitudes, Opt. Lett. 12, 614 (1987).

[39] M. N. Islam, C. D. Poole, and J. P. Gordon, Soliton trapping in birefringent optical fibers, Opt. Lett. 14, 1011 (1989).

[40] S. T. Cundiff, B. C. Collings, N. N. Akhmediev, J. M. Soto-Crespo, K. Bergman, and W. H. Knox, Observation of Polarization-Locked Vector Solitons in an Optical Fiber, Phys. Rev. Lett. 82, 3988 (1999).

[41] W. H. Renninger and F. W. Wise, Optical solitons in gradedindex multimode fibres, Nat. Commun. 4, 1719 (2013).

[42] Q.-F. Yang, X. Yi, K. Y. Yang, and K. Vahala, Stokes solitons in optical microcavities, Nat. Phys. 13, 53 (2017).

[43] J. K. Jang, A. Klenner, X. Ji, Y. Okawachi, M. Lipson, and A. L. Gaeta, Synchronization of coupled optical microresonators, Nat. Photon. 12, 688 (2018).

[44] E. Obrzud, S. Lecomte, and T. Herr, Temporal solitons in microresonators driven by optical pulses, Nat. Photon. 11, 600 (2017). 
[45] Y. Wang, F. Leo, J. Fatome, M. Erkintalo, S. G. Murdoch, and S. Coen, Universal mechanism for the binding of temporal cavity solitons, Optica 4, 855 (2017).

[46] J. K. Jang, M. Erkintalo, S. Coen, and S. G. Murdoch, Temporal tweezing of light through the trapping and manipulation of temporal cavity solitons, Nat. Commun. 6, 7370 (2015).

[47] J. P. Gordon and H. A. Haus, Random walk of coherently amplified solitons in optical fiber transmission, Opt. Lett. 11, 665 (1986).

[48] C. Bao, Y. Xuan, C. Wang, J. A. Jaramillo-Villegas, D. E. Leaird, M. Qi, and A. M. Weiner, Soliton repetition rate in a silicon-nitride microresonator, Opt. Lett. 42, 759 (2017).

[49] H. Lee, T. Chen, J. Li, K. Y. Yang, S. Jeon, O. Painter, and K. J. Vahala, Chemically etched ultrahigh-Q wedge-resonator on a silicon chip, Nat. Photon. 6, 369 (2012).
[50] A. M. Weiner, Femtosecond pulse shaping using spatial light modulators, Rev. Sci. Instr. 71, 1929 (2000).

[51] X. Yi, Q.-F. Yang, K. Y. Yang, and K. Vahala, Theory and measurement of the soliton self-frequency shift and efficiency in optical microcavities, Opt. Lett. 41, 3419 (2016).

[52] S. Coen, H. G. Randle, T. Sylvestre, and M. Erkintalo, Modeling of octave-spanning Kerr frequency combs using a generalized mean-field Lugiato-Lefever model, Opt. Lett. 38, 37 (2013).

[53] Y. K. Chembo and C. R. Menyuk, Spatiotemporal LugiatoLefever formalism for Kerr-comb generation in whisperinggallery-mode resonators, Phys. Rev. A 87, 053852 (2013).

[54] See Supplemental Material at http://link.aps.org/supplemental/ 10.1103/PhysRevA.103.L011501 for more details of the numerical simulations and discussion of asymmetric motion. 